\title{
Diseño e implementación de controladores digitales a través de un sistema de adquisición de datos
}

\author{
Design and implementation of digital controllers through a data acquisition \\ system. \\ Juan Pablo González Tamayo \\ Ingeniería Electrónica, Universidad Tecnológica de Pereira, Colombia \\ Correo-e: jpgonzalez@utp.edu.co
}

\begin{abstract}
Resumen- En este artículo se mostrarán los resultados de la implementación de diferentes controladores digitales, como el PID con transformación bilineal y el control por ubicación de polos mediante la realimentación de estados, en una planta identificada de segundo orden; para lo cual se emplea un sistema de adquisición de datos, que se programa desde el entorno de desarrollo Matlab.
\end{abstract}

Palabras clave - Adquisición de datos, control PID, control por realimentación de estados y asignación de polos, identificación de sistemas.

Abstract- This article will show the results of the implementation of different digital controllers, such as PID with bilinear transformation and pole placement control by state feedback, in a second order identified plant; for which a data acquisition system is employed, that is programmed from Matlab development environment.

Key Word - Control by state feedback and pole placement, data acquisition, PID control, system identification.

\section{INTRODUCCIÓN}

Con el advenimiento de la electrónica digital y el notable avance de los microprocesadores en los últimos años, se ha favorecido el procesamiento digital de señales, para dar solución a diferentes necesidades en el ámbito de las comunicaciones, electromedicina, automatización y control de procesos.

En el caso del control digital de sistemas dinámicos, se cuenta con la posibilidad de implementar algoritmos en sistemas embebidos o en computadores de propósito general a través de un sistema de adquisición de datos; éste último, caracterizándose por tener buenas prestaciones en cuanto a la potencia de procesamiento, capacidad de almacenamiento, conectividad, velocidad de medición, captura y monitoreo de datos en tiempo real.
Aprovechando los beneficios que ofrecen los sistemas de adquisición de datos, se procederá a diseñar e implementar en un sistema dinámico, dos de los controladores digitales más representativos del sector industrial y académico: el PID digital por aproximación trapezoidal, con modificaciones en su componente derivativa y el control por realimentación de estados y reubicación de polos, con seguimiento de referencia y observadores de estado.

En primera instancia se hará referencia a los sistemas de adquisición de datos y a la tarjeta multifunción NI-6024E que se empleará para la obtención de los diferentes resultados.

Para el adecuado diseño de los controladores, se realiza una identificación paramétrica de un sistema de segundo orden, emulado a través de un circuito con amplificadores operacionales. A partir de allí, se diseña e implementa (en tiempo real) en el lenguaje de programación Matlab, los controladores I-PD Y PI-D aproximados de forma trapezoidal; y un controlador con servo, regulación y estimación de estados. Finalmente se comparan y analizan las capturas obtenidas de la señal de referencia generada, la salida del sistema y la señal de control para cada uno de los algoritmos implementados.

\section{SISTEMA DE ADQUISICIÓN DE DATOS}

Como se menciona en [1], el propósito de cualquier sistema de adquisición de datos es proporcionar las herramientas y los recursos necesarios para medir y analizar fenómenos o procesos físicos. Por lo tanto, un sistema de este tipo puede considerarse como un conjunto de hardware y software que conectan al usuario con el mundo físico.

En la Figura 1 se muestra un diagrama que representa la arquitectura básica de un sistema de adquisición de datos. Por un lado la señal análoga es captada por un sensor, luego acondicionada, posteriormente convertida en bits que la computadora puede interpretar $\mathrm{y}$, por último, analizada en un 
software para extraer información significativa. Por otra parte, los datos digitales provenientes de la computadora se convierten en una señal analógica que es enviada a un actuador.

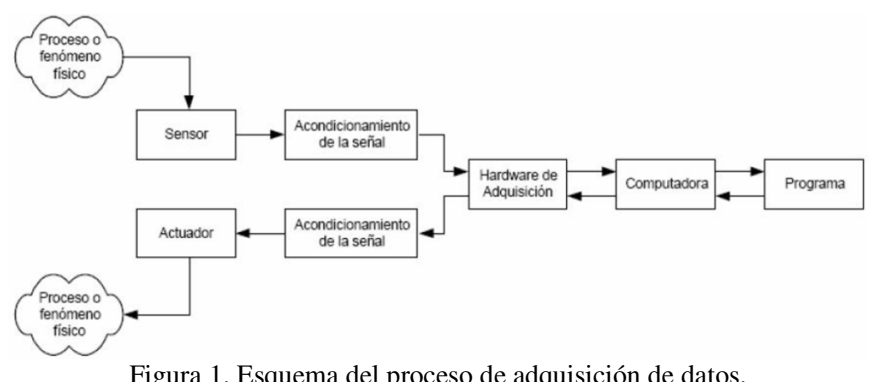

\section{A. Hardware de adquisición de datos}

El hardware de adquisición de datos puede ser interno o externo. En el primer caso, el mismo se instala en una ranura de expansión dentro de la computadora, en el otro, se conecta a la computadora a través de algún puerto como el USB, mediante un cable externo. En el nivel más simple, el hardware de adquisición de datos se caracteriza por los subsistemas que posee. Un subsistema es un componente del hardware de adquisición de datos que realiza una tarea especializada. Los subsistemas más comunes son:

1) Subsistema de entradas analógicas: El subsistema de entradas analógicas convierte en bits las señales recogidas por los sensores desde el mundo físico, de modo que puedan ser interpretadas por la computadora al muestrear y cuantizar las señales analógicas usando uno o más canales. Normalmente se trata de dispositivos con varios canales que ofrecen 12 o 16 bits de resolución.

2) Subsistema salidas analógicas: El subsistema de salidas analógicas convierte datos digitales almacenados en la computadora en señales analógicas con sentido físico. Opera de modo inverso a como lo hace el subsistema de entradas analógicas. Las tarjetas típicas de adquisición de datos poseen dos canales de salida con 12 bits de resolución, aunque también hay hardware especializado que puede tener varios canales analógicos de salida.

\section{B. Tarjeta de adquisición de datos NI-6024E}

Para realizar la adquisición y exportación de datos, se optó por utilizar el modelo PCI NI-6024E, debido a que es un dispositivo multifuncional de alto rendimiento. Incluye 16 entradas analógicas con máximo voltaje en modo común o diferencial de $\pm 10 V$, resolución de 16 bits (diferencial), máxima velocidad de muestreo de $250 \mathrm{kS} / \mathrm{s}, 2$ salidas analógicas con un máximo voltaje de $\pm 10 \mathrm{~V}$, resolución de 16 bits, máxima velocidad de muestreo de $833 \mathrm{kS} / \mathrm{s}$, E/S digital y temporizador de E/S. En la Figura 2 se puede visualizar la placa física.

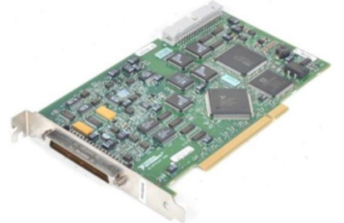

Figura 2. Tarjeta de adquisición de datos NI-6024E.

\section{IDENTIFICACIÓN DE SISTEMAS}

La identificación de sistemas tiene por objeto obtener el modelo de un sistema dinámico a partir de datos experimentales.

La obtención de un modelo a partir de datos experimentales conlleva las siguientes etapas fundamentales: la recolección de datos, la selección del modelo y la validación del modelo.

\section{A. Recolección de datos}

Los datos de entrada y salida se pueden obtener mediante un experimento diseñado específicamente para la identificación del sistema. En este caso, el usuario puede determinar que señales va a medir, cuándo y cómo las va a medir y también puede escoger las señales de entrada. El objetivo del diseño del experimento es entonces, seleccionar los datos que proporcionen la máxima información posible. En otros casos, el usuario no tiene la posibilidad de realizar el experimento, pero puede utilizar los datos obtenidos a partir de la operación normal del sistema y llevar a cabo con ellos la identificación del mismo.

\section{B. La Selección del Modelo}

Esta se realiza a partir de un grupo de modelos, eligiendo el más adecuado y representativo del sistema. Este paso es sin duda, el más importante y al mismo tiempo constituye la etapa más difícil en el procedimiento de la identificación. Es acá en donde el conocimiento previo del sistema y el de las características de cada modelo deben combinarse para obtener resultados satisfactorios. Algunas veces el modelo apropiado sólo se obtiene después de un cuidadoso proceso de modelado.

\section{Validación del Modelo}

La evaluación de la calidad del modelo se basa en determinar cómo se desempeña el modelo cuando se trata de reproducir con él los datos obtenidos en la medición experimental. Un comportamiento deficiente del modelo en este aspecto hace que el modelo sea rechazado, mientras que un buen desempeño, proporcionará cierta confianza en el modelo. Un modelo no se puede aceptar como la última y verdadera descripción del sistema; por el contrario, es mejor mirarlo sólo como una descripción suficientemente buena de ciertos aspectos que son de interés particular para un fin determinado [2]. 


\section{System Identification Toolbox de MATLAB}

El software System Identification Toolbox soporta el preprocesamiento de señales, la estimación y validación de modelos lineales a partir de datos en el dominio del tiempo y de la frecuencia. La información debe ser muestreada en instantes de tiempo discretos y espaciados uniformemente para obtener una secuencia de entrada:

$$
u=\{u(T), u(2 T) \ldots u(n T)\}
$$

y una secuencia de salida correspondiente:

$$
y=\{y(T), y(2 T) \ldots y(n T)\}
$$

$u(t)$ y $y(t)$ son los valores de la señal de entrada y de salida en el tiempo $t$, respectivamente. Este toolbox es compatible con el modelado tanto de uno o múltiples canales de entrada y salida de datos o de series de tiempo.

\section{E. Sistema dinámico de segundo orden}

Para realizar el proceso de identificación a través del System Identification Toolbox de MATLAB, se diseñó e implementó un sistema de segundo orden a través del uso de amplificadores operacionales. En la Figura 3 se muestra el diagrama esquemático y en la Figura 4 se muestra la respuesta simulada del sistema (Proteus 7 Professional) ante una entrada de referencia escalón unitario.

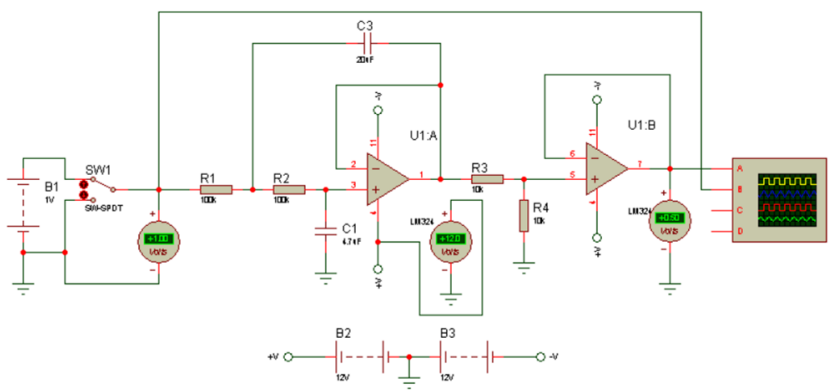

Figura 3. Diagrama esquemático del sistema de segundo orden.

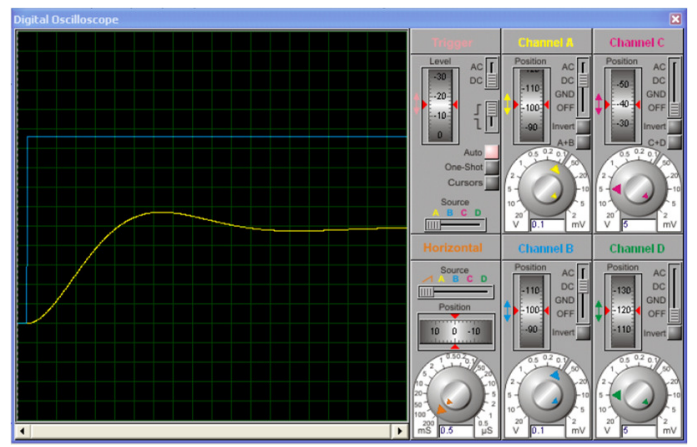

Figura 4. Simulación de la respuesta del sistema al escalón unitario.

Posteriormente a su implementación, se procede a excitar con una señal de $1 \mathrm{~V}$ por medio de la fuente de alimentación $D C$, obteniendo los siguientes parámetros de respuesta transitoria del sistema:

-Respuesta subamortiguada.

-Máximo sobreimpulso $M_{p}=20 \%$.

-Tiempo de establecimiento aproximado $t_{s}=11 \mathrm{~s}$.

-Ganancia de estado estacionario $K=0,5$.

-Sin retardo.

\section{F. Implementación y resultados}

En aras de obtener la recolección de datos para el proceso de identificación, se decide excitar el sistema con un tren de pulsos con $50 \%$ de ciclo de trabajo, con un periodo mayor o igual a 2 veces el tiempo de establecimiento del sistema en lazo abierto y múltiplo del periodo de muestreo a emplear en el proceso de adquisición de datos. Dado que el $t_{s}=11 \mathrm{~s}$ y el periodo de muestreo es $0.2 \mathrm{~s}$, entonces el periodo del pulso con el que se excitó la planta es $T_{\text {tren }}$ pulsos $=4 \times t s=44 \mathrm{~s}$, lo que cumpliría las condiciones para una adecuada recolección de datos.

Se realiza la adquisición y almacenamiento de los datos para su respectivo procesamiento, a través de 2 entradas análogas de la tarjeta de adquisición, por un tiempo de 4 veces el periodo del tren de pulsos. Es decir, $t_{a d q}=4 \times T_{\text {tren pulsos }}=176 \mathrm{~s}$.

Después de realizada la adquisición de la señal de excitación a la planta (tren de pulsos) y la respuesta de la misma, se almacenan en un arreglo de datos a través de una variable en un script de MATLAB, con el fin de realizar el proceso de identificación del modelo con la ayuda del GUI del System Identification Toolbox.

En primera instancia, se importaron las variables que contenían los datos de entrada y salida de la planta por medio de la opción Import data. A partir de allí se realiza el acondicionamiento de las señales a través de la función Prepocess, que consiste en eliminar los valores medios, particionar los datos (50\% para estimación y 50\% para validación), eliminar voltajes de offset y filtrar digitalmente.

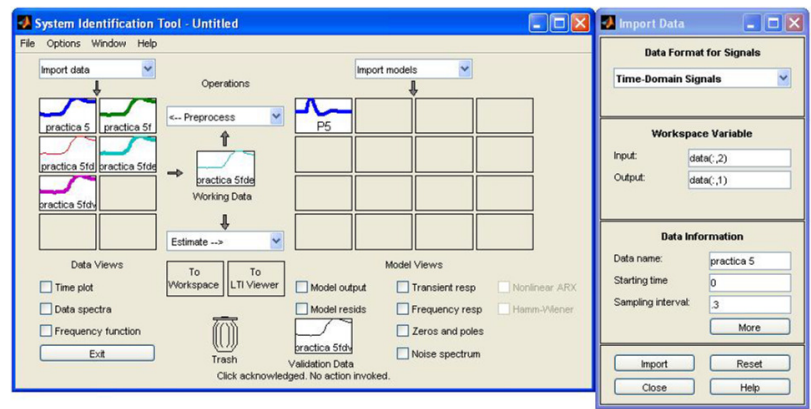

Figura 5. Entorno de trabajo del System Identification Toolbox.

Los datos previamente acondicionados y dispuestos para la estimación, fueron trasladados al Working Data del ident, y como paso a seguir se seleccionó la opción Process Models del menú desplegable Estimate; allí se configuró la estimación con dos polos subamortiguados, sin retardo y sin ceros. 
Posteriormente se realizó la validación del modelo estimado, obteniéndose en la Figura 6 la respuesta del sistema identificado; con una aproximación del $95,75 \%$, es decir, un error del $4,25 \%$ del sistema estimado con respecto al modelo real.

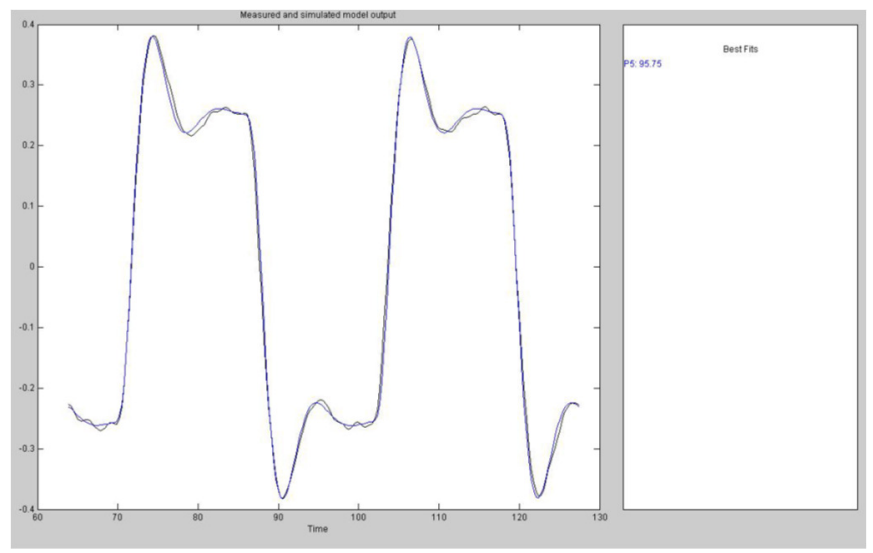

Figura 6. Validación de la identificación.

Desde el Workspace de Matlab se ejecutó el comando zpk y se obtuvo la función de transferencia:

$$
H(s)_{\text {ident }}=\frac{0,34341}{s^{2}+0,6654+0,6754}
$$

Como se puede apreciar el proceso tuvo resultados satisfactorios en todas sus etapas, puesto que el modelo que describe la dinámica del sistema, tiene una alta correspondencia con los datos medidos.

\section{IV.CONTROLADORES PID}

El controlador proporcional, integral y derivativo, o más comúnmente PID es el más popular en la práctica industrial. Es ampliamente conocido debido a la gran cantidad de montajes y la simplicidad en su uso, siendo un dispositivo de control genérico donde el diseñador sólo tiene que dar valores adecuados, según lo requiera la situación, a los distintos parámetros que contiene [3]. Mientras que el controlador proporcional siempre se utiliza, se tiene la opción de elegir entre una o ambas de las otras dos maniobras. La forma estándar del controlador PID se muestra en la Figura 7.

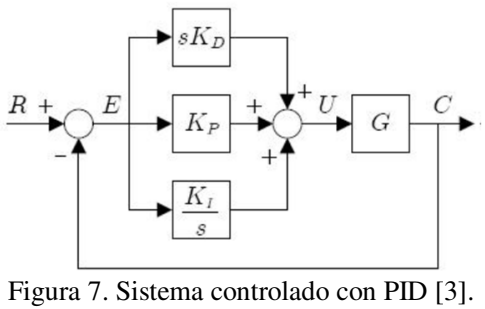

La expresión de la señal de control en el dominio de la frecuencia:

$$
U(s)=\left(K_{P}+\frac{K_{I}}{s}+s K_{D}\right) E(s)
$$

\section{A. Modificaciones del PID}

El sistema de control PID básico presenta inconvenientes, si se presenta alguna de las siguientes situaciones:

-Si existe un cambio repentino en el setpoint (punto de ajuste) como lo suele haber ante una entrada de referencia tipo función escalón, debido a la presencia del término derivativo en la acción de control, la señal de control $u(t)$ contendrá una función impulso (una función delta) ya que, en el momento del cambio finito de la referencia, la derivada del error se hace infinita, por lo que la actuación tambíen se hace muy grande teóricamente infinita-. El gran cambio introducido por el modo derivado se conoce como "derivative kick" o reacción derivativa.

-El modo proporcional introduce grandes saltos en la señal de control, debido al primer sobreimpulso de la salida ante cambios bruscos de la referencia. El cambio abrupto inducido por la acción proporcional es llamado "proportional kick" o reacción proporcional.

Para evitar el fenómeno de la reacción del punto de ajuste, la expresión estándar del PID se suele modificar para obtener mejores prestaciones. En los siguientes apartados se explican algunas de las mejoras más habituales.

1) Control PI-D: Una forma de solucionar este problema es hacer que la parte derivativa del PID no actúe sobre la señal del error $E(s)$, sino exclusivamente sobre la señal de salida $C(s)$ del sistema. Esta estrategia no altera el comportamiento del sistema controlado ante una entrada escalón y sólo suprime los impulsos infinitos que introduce la derivación del salto finito. Estos impulsos infinitos producirían una especie de golpes bruscos en el sistema: es el efecto kick-off que evita esta estrategia [3].

En la Figura 8 se ilustra la estrategia descrita.

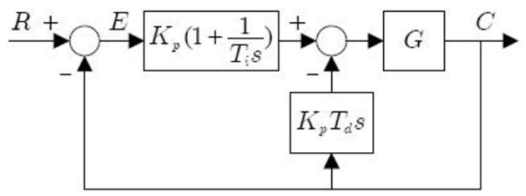

Figura 8. Controlador PI-D [3].

La señal de control obtenida en el dominio del tiempo:

$$
u(t)=k_{p} e(t)+k_{i} \int e(\tau) d \tau-k_{d} \frac{d y(t)}{d t}
$$

2) Control I-PD: Tanto el control PID como el control PI-D implican una función escalón en la señal $u(t)$. En muchas 
ocasiones, tal cambio escalón en la señal de control tal vez no sea conveniente. Por tanto, puede convenir ajustar la acción proporcional y la acción derivativa a la trayectoria de realimentación, a fin de que estas acciones sólo afecten la señal de realimentación $C(s)$ [4]. A ésta estrategia se le conoce como controlador I-PD y se muestra en la Figura 9.

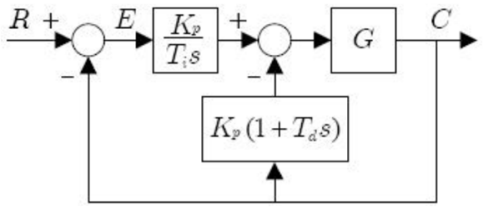

Figura 9. Controlador I-PD [3].

La expresión de la señal de control que se obtiene:

$$
u(t)=k_{p} y(t)+k_{i} \int e(\tau) d \tau-k_{d} \frac{d y(t)}{d t}
$$

\section{B. Discretización del PID}

1) Control Proporcional: Este tipo de controlador genera una salida que es proporcional al error actuante [2]. La ecuación de un controlador proporcional continuo está dada por:

$$
m(t)=k_{p} e(t)
$$

La forma discreta de la ecuación 7 es:

$$
m(k T)=k_{p} e(k T)
$$

Donde:

$$
e(k T)=r(k T)-y(k T)
$$

Además $e(k)$ se define como el error de seguimiento, $y(k)$ la salida del sistema controlado y $r(k)$ la referencia.

2) Control Integral: El proceso de integración puede ser representado gráficamente como en la Figura 10:

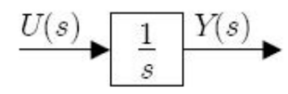

Figura 10. Operación integral [3].

Existen diversos métodos para aproximar la integral entre dos periodos de muestreo. El método bilineal, trapezoidal o de Tustin define la integral entre dos periodos de muestro como el trapecio que forman el valor actual de la función y el anterior, tal como se muestra en la Figura 11.

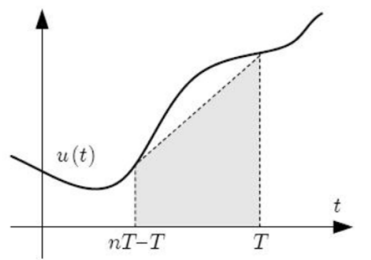

Figura 11. Aproximación trapezoidal [3].

Matemáticamente se puede expresar como:

$$
\int_{k T-T}^{k t} u(\tau) d \tau=T \frac{k(n T)+u(k T-T)}{2}
$$

De esta forma, la función integral se puede completar como:

$$
y(k T)=y(k T-T)+T \frac{u(k T)+u(k T-T)}{2}
$$

3) Control Derivativo: El proceso de derivación se puede representar como en la Figura 12:

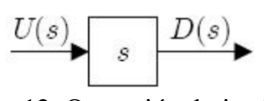

Figura 12. Operación derivada [3].

Al igual que en el caso integral, la derivada de una función se puede aproximar por diferentes métodos. El método de diferencia hacia atrás, define la derivada como el último cambio de la función dividido entre el tiempo que ha transcurrido, tal como se muestra en la Figura 13.

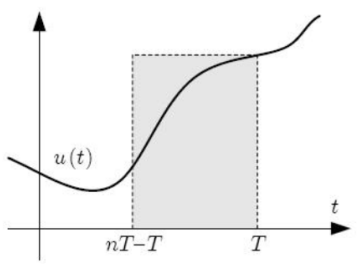

Figura 13. Aproximación por diferencia hacia atrás [3].

En términos de ecuaciones en diferencia, donde $d(k T)$ es la derivada de $u(k T)$ :

$$
d(k T)=\frac{u(k T)-u(k T-T)}{T}
$$

4) Controlador PID digital: A partir de la combinación de cada una de las aproximaciones discretas anteriores, es posible obtener una señal de control de la siguiente manera:

$$
u(k)=k_{p} e(k)+k_{i} u_{i}(k)-k_{d} \frac{e(k)-e(k-1)}{T}
$$

Donde:

$$
u_{i}(k T)=u_{i}(k T-1)+T \frac{e(k)+e(k-1)}{2}
$$




\section{Algoritmo de un controlador digital}

La implementación de un controlador digital, requiere iterar cada $T$ segundos (periodo de muestreo) el siguiente algoritmo:

1. Se inicializan los valores pasados de la señal de error $e(k-$ 1), la respuesta de la planta $y(k-1)$ y la señal de control $u(k-$ 1) si es necesario.

2. Se inicia un ciclo o bucle.

3. Se define la referencia $r(k)$.

4. La tarjeta de adquisición toma el valor de la salida del sistema a regular en el instante actual, es decir, el valor de $y(k T)$.

5. Se calcula el error para el instante actual $k T$, como la diferencia de la referencia y la salida del sistema, $e(k T)=$ $r(k T)-y(k T)$.

6. Se calcula la acción de control para el instante actual, es decir $u(k T)$

7. Se satura $u(k T)$ según el rango máximo permitido en el canal de salida análogo de la tarjeta de adquisición.

8. La acción de control $u(k T)$ es exportada a través de la salida análoga de la tarjeta de adquisición.

9. Se actualizan los valores pasados de las variables con los del instante actual.

10. Se genera una pausa de un tiempo de muestreo $T$.

11. Se finaliza el ciclo o bucle.

\section{Implementación y resultados}

A través de un script en MATLAB, se procedió a diseñar e implementar un controlador PID digital de manera que el sistema controlado respondiera a los siguientes criterios de diseño:

Tiempo de establecimiento $t_{s}=4 \mathrm{~s}$.

Coeficiente de amortiguamiento $\zeta=0,7$.

Tercer polo ubicado en $s=-2 \omega_{n}$.

Al ejecutar el script se calcularon las constantes del controlador PID: $K_{p}=20,61, k_{i}=16,98$ y $k_{d}=12,21$ en el dominio de Laplace, a través de la comparación de coeficientes de los denominadores en lazo cerrado del sistema-controlador y el polinomio deseado, de acuerdo a las características transitorias mencionadas anteriormente.

Además se configuraron los canales de entrada y salida análoga en la tarjeta de adquisición, para obtener la señal de la salida de la planta $y(k)$ y enviar la señal de control $u(k)$ hacia la entrada de la misma, con base en el error que se genera entre la señal de referencia $r(k)$ (Figura 14) y la salida del sistema.

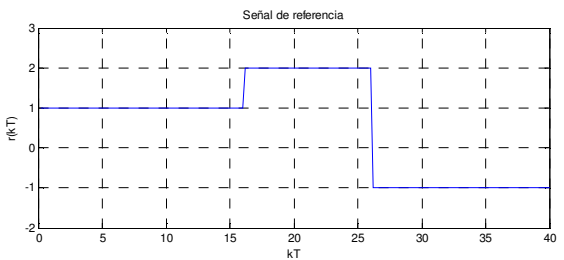

Figura 14. Señal de referencia $r(k)$ generada en Matlab.

1) Controlador I-PD: Dadas las ecuaciones discretas discutidas anteriormente, se efectuaron los ajustes necesarios para obtener el control I-PD discreto. Al implementar el controlador a través del sistema de adquisición de datos, se obtuvieron los resultados que se aprecian en la Figura 15.
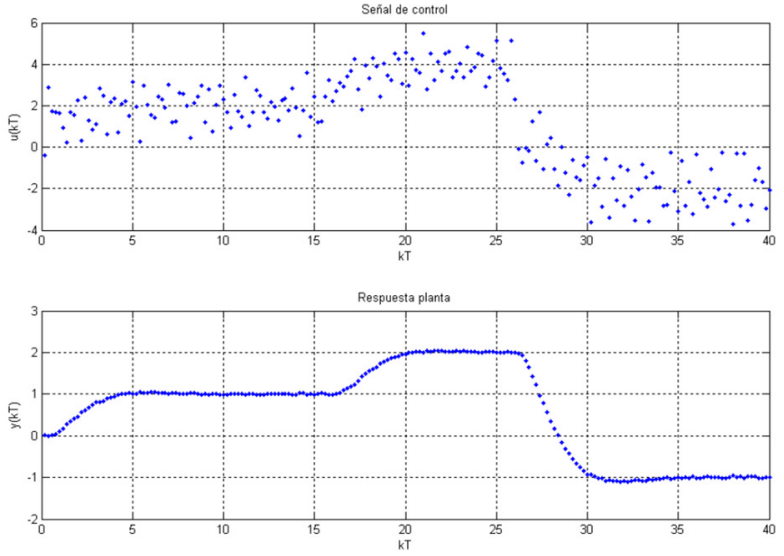

Figura 15. Señales $y(k T)$ y $u(k T)$ adquiridas en Matlab.

2) Controlador PI-D: Realizando los ajustes necesarios en la ecuación de diferencias de la señal de control, se obtuvieron los resultados que se muestran en la Figura 16.
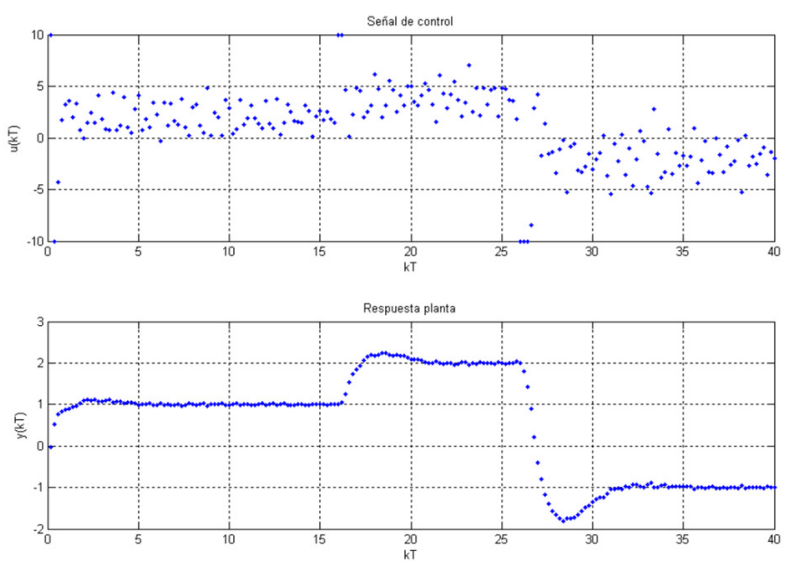

Figura 16. Señales $y(k T)$ y $u(k T)$ adquiridas en Matlab.

Después de implementar las modificaciones del controlador PID digital y al analizar las gráficas de la respuesta de la 
planta en cada caso, se infiere que el mejor rendimiento se obtuvo con el control I-PD, ya que la planta tuvo un tiempo de establecimiento alrededor de $4 s$ y presentó un sobrepaso casi imperceptible.

La implementación PI-D presentó problemas de saturación con la señal de control $u(k)$ en los instantes que la referencia $r(k)$ tuvo transiciones abruptas, además se observaron sobreimpulsos considerables en la respuesta del sistema, lo que no es pertinente a la hora de controlar procesos industriales que poseen estrictas restricciones de operación.

\section{CONTROL POR REALIMENTACION DE ESTADOS Y ASIGNACIÓN DE POLOS}

Para este esquema de control según [2], el problema de diseño consiste en determinar un algoritmo que permita generar una secuencia de valores de la variable de control $u(k)$ de la planta, de manera que las salidas $y(k)$ cumplan con las especificaciones de funcionamiento establecidas, como por ejemplo:

-Atenuación de las perturbaciones en la carga.

-Reducción del efecto del ruido en la medida.

-Seguimiento de la señal de comando.

-Variaciones e incertidumbre en el comportamiento del proceso.

\section{A. Análisis de sistemas de control en el espacio de estado}

Para los sistemas lineales de tiempo discreto invariantes en el tiempo, la ecuación de entrada y la ecuación de salida se pueden escribir:

$$
\begin{gathered}
x(k+1)=A x(k)+B u(k) \\
y(k)=C x(k)+D u(k)
\end{gathered}
$$

En donde:

$A=$ Matriz de estado (matriz $n \times n$ )

$B=$ Matriz de entrada (matriz $n \times r)$

$C=$ Matriz de salida (matriz $m \times n$ )

$D=$ Matriz de transmisión directa (matriz $m \times r$ )

En la representación por variable de estados de un sistema lineal, las matrices $A$ y $C$ describen el comportamiento no forzado del sistema (o el comportamiento a entrada-cero), mientras que la matriz $B$ caracteriza el efecto de la entrada (o el control) sobre la dinámica del sistema. La matriz $D$ representa la transmisión directa de la entrada a la salida.

El sistema se puede representar como se muestra en la Figura 17 , en donde $u(k)$ es el vector de entrada, $y(k)$ es el vector de salida y $x(k)$ es el vector de estado.

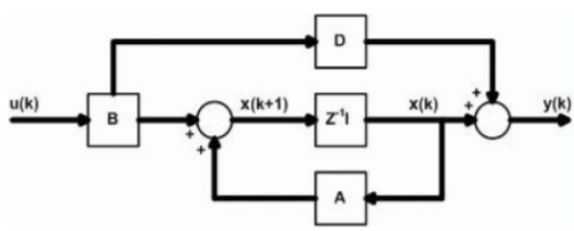

Figura 17. Diagrama en bloques de un sistema de tiempo discreto lineal e invariante en el tiempo [2].

\section{B. Controlabilidad}

Se dice que un sistema es completamente controlable, si existe una señal de control $u(k T)$, no restringida, definida a lo largo de un número finito de periodos de muestreo de manera que, partiendo de cualquier estado inicial, el estado $x(k T)$ pueda ser transferido al estado deseado $x_{f}$ en $n$ periodos de muestreo como máximo [2].

Entonces el sistema descrito es controlable si:

$$
\text { Rango }\left[B A B A^{2} B \cdots A^{n-1} B\right]=n
$$

\section{Observabilidad}

Se dice que un sistema es complemente observable si cualquier estado inicial $x(0)$ puede determinarse a partir de la observación de $y(k)$ en $n$ períodos de muestreo como máximo.

La condición suficiente y necesaria para que un sistema sea completamente observable es [2]:

$$
\text { Rango }\left[C^{\prime} A^{\prime} C^{\prime} A^{2} C^{\prime} \cdots\left(A^{\prime}\right)^{n-1} C^{\prime}\right]=n
$$

\section{Reubicación de polos mediante la realimentación de estados}

El método de asignación de polos, comienza con la determinación de los polos de lazo cerrado deseados, utilizando para ello especificaciones basadas en la respuesta transitoria y/o en los requerimientos de respuesta en frecuencia. Si se desea ubicar los polos de lazo cerrado en $z=$ $z_{1}, z=z_{2}, \ldots z=z_{n}$ es posible elegir una matriz de ganancia de realimentación $K$ adecuada, que force al sistema a tener los polos de lazo cerrado en el lugar deseado siempre y cuando el sistema sea de estado completamente controlable y completamente observable.

Si se elige como ley de control:

$$
u(k)=-K x(k)
$$

Se obtiene el sistema de control realimentado mostrado en la Figura 18. 


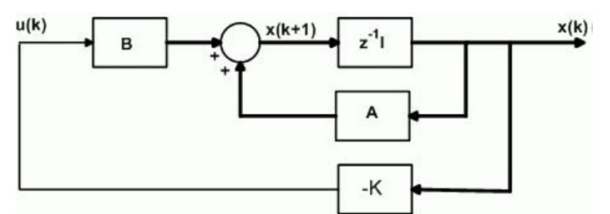

Figura 18. Sistema de control con realimentación de estados [2].

La matriz $K$ se llama "matriz de ganancia de realimentación" y convierte al sistema en un sistema de control en lazo cerrado, cuya dinámica queda determinada por las especificaciones de funcionamiento dadas, las cuales determinan a la vez la ubicación de los polos de lazo cerrado deseados.

La ecuación característica del sistema en lazo cerrado es:

$$
|z I-A+B K|=z^{n}+\alpha_{1} z^{n-1}+\alpha_{2} z^{n-2} \cdots+\alpha_{n-1} z+\alpha_{n}
$$

En donde $\alpha_{1}, \alpha_{2}, \ldots \alpha_{n}$ son los coeficientes de la ecuación característica deseada.

La fórmula de Ackerman permite calcular directamente la matriz de ganancia de realimentación, a partir de la ecuación [2]:

$$
K=\left[\begin{array}{llll}
0 & 0 & \cdots & 1
\end{array}\right]=\left[B A B A^{2} B \cdots A^{n-1} B\right]^{-1} \phi(A)
$$

En donde:

$$
\phi(A)=z^{n}+\alpha_{1} z^{n-1}+\alpha_{2} z^{n-2} \cdots+\alpha_{n-1} z+\alpha_{n}
$$

Que se obtiene de:

$$
\left(z-z_{1}\right)\left(z-z_{2}\right) \cdots\left(z-z_{n}\right)=z^{n}+\alpha_{1} z^{n-1}+\alpha_{2} z^{n-2} \cdots+\alpha_{n-1} z+\alpha_{n}=0
$$

\section{E. Sistema de control con realimentación de estado y entrada de referencia}

El sistema de control descrito anteriormente no tiene entrada de referencia y se denomina sistema de control tipo regulador. En la mayoría de los casos, es necesario que la salida $y(k)$ siga a una entrada de referencia $r(k)$, este sistema se denomina sistema de control tipo servo y su configuración básica se muestra en la Figura 19.

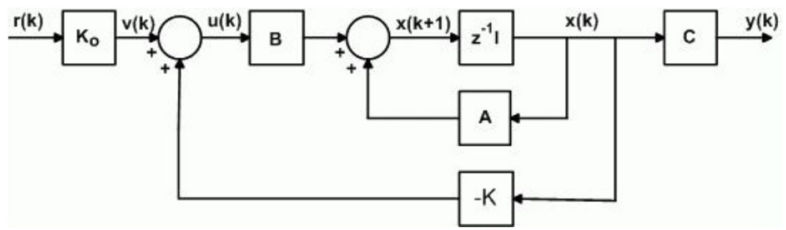

Figura 19. Sistema de control con entrada de referencia [2].

La señal de control está dada por:

$$
u(k)=K_{0} r(k)-K x(k)
$$

En donde $K_{0}$ es una constante que se debe determinar y $r(k)$ es la entrada de referencia.

La introducción de la matriz de ganancia de realimentación $K$ modifica la ecuación característica del sistema original y al hacerlo, modifica también la ganancia de estado estable del sistema en lazo cerrado. En estas condiciones, la constante $K_{0}$ se puede tomar como un parámetro de ajuste en el circuito del set-point, tal que el valor de la respuesta en régimen permanente del sistema ante un escalón unitario sea igual a la unidad, es decir, tal que $y(\infty)=1$. Al aplicar el teorema del valor final se cumple que:

$$
K_{0} \lim _{z \rightarrow 1} C[z I-A+B K]^{-1} B=1
$$

La ecuación 25 permite calcular el valor adecuado de $K_{0}$ para que el error de estado estable del sistema en lazo cerrado ante una entrada en escalón unitario, aplicada en la señal de referencia, sea igual a cero [2].

\section{F. Observadores de estado de orden completo}

En la práctica, no todas las variables de estado de un sistema se pueden medir en forma directa. Este hecho hace necesario estimar el valor de aquellas variables de estado cuya medición directa no es posible. La estimación se debe realizar a partir de mediciones en las variables de entrada y en las variables de salida.

Si el estado $x(k)$ debe estimarse, es necesario que el estado estimado $q(k)$ y el estado real $x(k)$ sean iguales o lo más aproximadamente iguales.

La Figura 20 representa el sistema de control con la matriz de ganancia de realimentación $K$ y el observador de estado tipo predictor. La matriz $L$ se denomina matriz de ganancia de realimentación del observador.

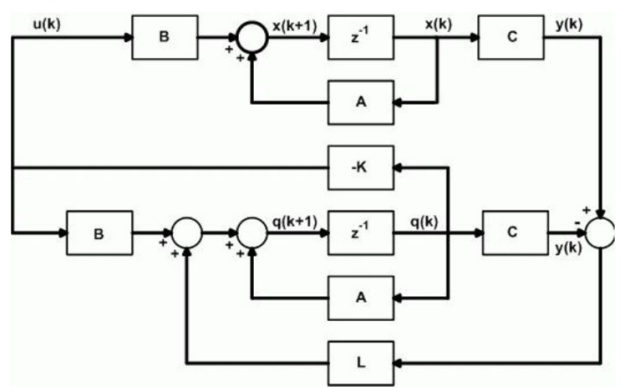

Figura 20. Sistema de control con realimentación del estado observado [2].

La ecuación del observador tipo predictor de orden completo se puede escribir en la forma:

$$
q(k+1)=(A-L C-B K) q(k)+L y(k)
$$

La matriz $L$ es una matriz pesante y se debe diseñar de modo que $q(k)$ se aproxime asintóticamente a $x(k)$ cuando $k \rightarrow \infty$. Es 
decir, para que la dinámica del error sea estable y que $e(k)$ llegue a cero con suficiente rapidez.

Los polos de lazo cerrado deseados para el observador se diseñan de manera que el sistema cumpla con los requisitos de funcionamiento especificados y se eligen de modo que su respuesta sea de dos a cuatro veces más rápida que la del sistema. La matriz de ganancia del observador se calcula a través de la fórmula de Ackerman a partir de la ecuación:

$$
L=\phi(A)\left[\begin{array}{c}
C \\
C A \\
C A^{2} \\
\vdots \\
C A^{n-1}
\end{array}\right]^{-1}\left[\begin{array}{c}
0 \\
0 \\
0 \\
\vdots \\
1
\end{array}\right]
$$

En donde:

$$
\phi(A)=A^{n}+\alpha_{1} A^{n-1}+\alpha_{2} A^{n-2} \cdots+\alpha_{n-1} A+\alpha_{n} I
$$

Siendo $\alpha_{1}, \alpha_{2}, \ldots \quad \alpha_{n}$ los coeficientes de la ecuación característica deseada para el observador:

$$
\left(z-p_{1}\right)\left(z-p_{2}\right) \cdots\left(z-p_{n}\right)=z^{n}+\alpha_{1} z^{n-1}+\alpha_{2} z^{n-2} \cdots+\alpha_{n-1} z+\alpha_{n}
$$

Por lo tanto, el diseño de la matriz de ganancia de realimentación $K$ y el diseño de la matriz de ganancia del observador $L$, son dos problemas independientes entre si que se combinan para obtener el sistema de control con realimentación del estado observado [2].

\section{G. Implementación y resultados}

De acuerdo al planteamiento teórico realizado, se diseña un regulador, un observador de estados y un servo para el seguimiento de una señal de referencia, que mejore el tiempo de establecimiento y el máximo sobreimpulso de la planta de segundo orden en lazo abierto, teniendo precaución de no sobrepasar el limite de $\pm 10 \mathrm{~V}$ en la señal de control $u(k)$.

Los polos para la matriz de ganancia de realimentación $K$ se asignaron del polinomio característico de un sistema de segundo orden, con coeficiente de amortiguamiento $\zeta=0,7 \mathrm{y}$ un tiempo de establecimiento $t_{s}=4 \mathrm{~s}$. El observador es de tipo predictor dead beat, es decir, que los polos de la matriz $L$ están ubicados en el origen del plano $z$ (coordenadas 0,0). Las matrices $L$ y $K$ se calcularon usando la formula de Ackerman.

Se desarrolla el código necesario en un script de Matlab para calcular las constantes mencionadas en el párrafo anterior, además de la configuración de los canales de entrada y salida analógica del dispositivo de adquisición, a través de los cuales se obtienen las muestras de la salida del sistema y la exportación de la señal de control, que deberá responder a la señal de referencia del sistema, tal y como se muestra en la Figura 21.

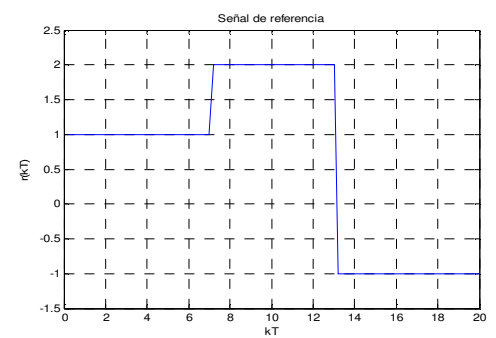

Figura 21. Señal de referencia $r(k)$ generada en Matlab.

Al ejecutar el programa y al asignarle valores nulos a las condiciones iniciales de las variables de estado, se obtuvo la señal de control y la respuesta de la planta (Figura 22), durante la ejecución del controlador en 20 segundos (100 muestras).
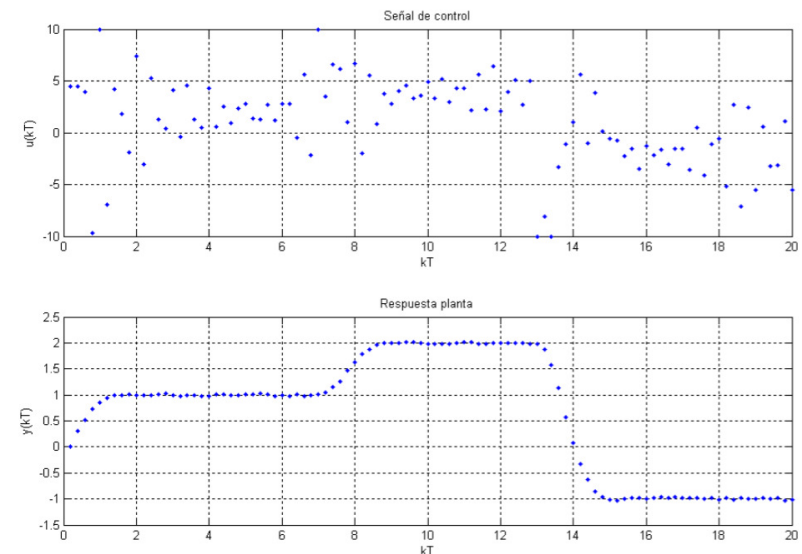

Figura 22. Señales $u(k T)$ y $y(k T)$ adquiridas del sistema, sin condiciones iniciales.

Al considerar el efecto de perturbaciones externas, se establecieron valores iniciales al vector de estados para analizar el efecto causado en la respuesta del controlador y en la salida de la planta. Al asignar $x(0)=\left[\begin{array}{ll}0,5 & 0,3\end{array}\right]^{\prime}$, el desempeño del sistema realimentado se observa en la Figura 23.
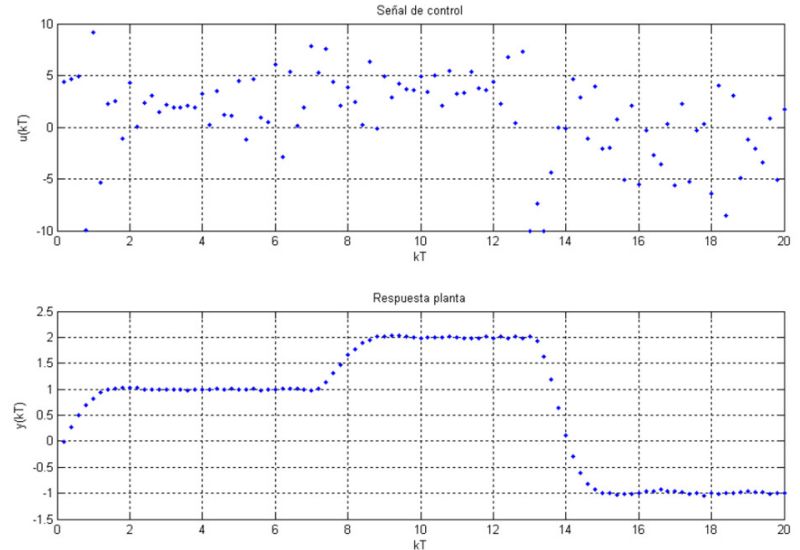

Figura 23. Señales $u(k T)$ y $y(k T)$ adquiridas del sistema, con condiciones iniciales. 
Se observa que tanto con o sin condiciones iniciales, el regulador, el servo y el estimador de estados llevaron la respuesta de la planta al nivel indicado por la referencia en menos de $4 s$ y con un sobrepaso aproximado al $0 \%$.

\section{CONCLUSIONES}

Para que el control digital de un proceso en tiempo real sea realizable, debe disponerse de un sistema embebido o un computador de buen desempeño, que esté dotado de una tarjeta de adquisición de datos. Esta última opción ofrece ventajas en cuanto a diversidad y flexibilidad en el uso del software de programación, la simplicidad del manejo del hardware del mismo, la visualización y monitoreo de las señales de interés. Como desventaja, se puede evidenciar el alto costo comercial de las tarjetas de adquisición de datos y de los computadores de propósito general de alto rendimiento.

Como paso previo al diseño de controladores, es sugerente obtener el modelo paramétrico o no paramétrico del proceso en el punto de operación requerido. En este caso se realizó la identificación del sistema a través del System Identification Toolbox de MATLAB, como el software de apoyo para la estimación fuera de línea del modelo, ya que esta aplicación cumple con las herramientas apropiadas para realizar un análisis y preprocesamiento de los datos antes de realizar la identificación, además de los variados métodos para realizar la estimación y validación del sistema obtenido.

Bajo las mismas condiciones de diseño, el controlador I-PD mostró un mejor desempeño que el controlador PI-D, ya que se obtuvieron las características transitorias requeridas del sistema, sin evidenciar algún tipo de saturación en la señal de control.

Los resultados obtenidos con el regulador, servo y estimador de estados, muestran el buen desempeño del controlador, ya que se consiguió la respuesta transitoria requerida del sistema con o sin perturbaciones. Aunque se observó que la señal de control se saturó en las transiciones abruptas de la señal de referencia.

Al observar los resultados de las implementaciones anteriores, es posible discernir que los controladores PID y por realimentación de estados son estrategias de control con muy buenas prestaciones, de bajo costo computacional y de relativa facilidad de implementación. A diferencia del PID, el control por realimentación de estados, permite resolver problemas de servo y regulación, siempre y cuando el sistema sea completamente controlable y completamente observable.

\section{REFERENCIAS}

[1] MathWorks, Data acquisition toolbox. User's Guide, Massachusetts, 2012.

[2] L. E. García Jaimes, "Control digital: teoría y práctica", 2 ed., Medellín: Politécnico Colombiano Jaime Isaza Cadavid, 2009, p. 412.

[3] J. J. Gil Nobajas y A. R. Díaz Cordovés, "Fundamentos de control automático de sistemas continuos y muestreados", San Sebastián: Universidad de Navarra, 2009, p. 215.

[4] K. Ogata, "Ingeniería de control moderna", 5 ed., Madrid: Pearson Educación, 2010, p. 894.

[5] K. Ogata, "Sistemas de control en tiempo discreto", 2 ed., México: Prentice Hall, 2000, p. 745.

[6] N. I. Corporation, 6024E user manual, Austin, Texas, 2000 .

[7] K. J. Astrom y B. Wittenmark, "Computer-controlled System: theory and design", 3 ed., New Jersey: Prentice Hall, 1996, p. 557.

[8] K. M. Moudgalya, "Digital control", Chichester: John Wiley and Sons, 2007, p. 543. 